\title{
Vagal Regulation of Respiratory Clocks in Mice
}

\author{
Hideki Bando, ${ }^{1,2}$ Takeshi Nishio, ${ }^{1,2}$ Gijsbertus T. J. van der Horst, ${ }^{3}$ Satoru Masubuchi, ${ }^{1}$ Yasuo Hisa, ${ }^{2}$ Hitoshi Okamura ${ }^{1}$ \\ ${ }^{1}$ Division of Molecular Brain Science, Department of Brain Sciences, Kobe University Graduate School of Medicine, Chuo-ku, Kobe 650-0017, Japan, \\ ${ }^{2}$ Department of Otolaryngology, Kyoto Prefectural University of Medicine, Kamikyo-ku, Kyoto 602-8566, Japan, and ${ }^{3}$ Department of Genetics, Erasmus \\ University Medical Center, 3000 CA Rotterdam, The Netherlands
}

The present study addresses the role of the circadian system in day-night changes of respiratory functions in the mouse. In all airway tissues investigated (i.e., larynx, trachea, bronchus, and lung), we observed clear rhythmic expression of the Per1, Per2, Bmal1, and Clock core oscillator genes (the latter two genes oscillating in antiphase with the Per genes), as well as the clock-regulated $D b p$ gene. Oscillations were abolished in arrhythmic $\mathrm{Cry} 1^{-/-} \mathrm{Cr} y 2^{-/-}$knock-out mice and after lesioning of the master clock in the suprachiasmatic nucleus (SCN) in wild-type animals. These findings indicate that respiratory system cells contain a functional peripheral oscillator that is controlled by the SCN. Furthermore, we found that the muscarinic acetylcholine receptor genes Chm2, Chm3, and Chm4 are expressed in a circadian manner, and that mucin secretion (rather than synthesis) by the airway submucosal glands is under circadian control. Signals from the SCN are mainly transmitted by the vagal nerve because unilateral vagotomy completely abolished rhythms in mucin and PER2 protein levels in the (operated) ipsilateral side of the submucosal glands, but not in the (intact) contralateral side. Thus, peripheral clock mediated circadian expression of muscarinic acetylcholine receptor proteins, and parasympathetic signaling between SCN and respiratory tissues are essential gears in conferring circadian "time" information to airway glands.

Key words: circadian; vagal nerve; clock genes; mucin; PER2; muscarinic receptor

\section{Introduction}

The mammalian circadian system orchestrates near 24 h rhythms in behavior and physiology. Accordingly, respiratory functions as well as many of the symptoms of airway diseases are associated with specific times of the day (Barnes, 1985). For example, hoarseness tends to occur in the early morning, whereas worsening of wheezing in asthmatic patients frequently occurs at midnight. Although mechanisms appear complex, it has been speculated that autonomic innervation of airway tissues through the vagal nerve plays a critical role in the circadian manifestation of respiratory symptoms (Nadel and Barnes, 1984). For instance, electrical stimulation of vagal nerves causes bronchoconstriction and mucus secretion, whereas muscarinic antagonists inhibit these processes. Nevertheless, there are no basic studies addressing the role of the vagal nerve in the circadian appearance of airway symptoms.

The understanding of the mammalian circadian system profoundly progressed in recent years. The previous molecular dissection of the biological clock has revealed that circadian oscillations are generated by a molecular oscillator, composed of interacting positive and negative transcription/translation feedback loops of a set of clock genes (Hastings et al., 2003). This

Received Sept. 21, 2006; revised March 10, 2007; accepted March 10, 2007.

This work was supported in part by The Scientific Grant of the 21st Century Centers of Excellence Program and The Special Promotion Funds from the Ministry of Education, Culture, Sports, Science, and Technology of Japan. We thank Dr. Toshio Terashima for discussion and Tsuyoshi Waku and Daisuke Matsui for their assistance.

Correspondence should be addressed to Dr. Hitoshi Okamura, Division of Molecular Brain Science, Department of Brain Sciences, Kobe University Graduate School of Medicine, 7-5-1 Kusunoki-cho, Chuo-ku, Kobe 650-0017, Japan. E-mail: okamurah@med.kobe-u.ac.jp.

DOI:10.1523/JNEUROSCI.4131-06.2007

Copyright $\odot 2007$ Society for Neuroscience $\quad$ 0270-6474/07/274359-07\$15.00/0 molecular core oscillator is present in most cells in the body and it is speculated that the mammalian circadian system consists of multiple oscillatory systems with a hierarchical architecture in which the "master" clock in the hypothalamic suprachiasmatic nucleus (SCN) drives "slave" oscillators in peripheral tissues (Schibler and Sassone-Corsi, 2002). In this circadian system, the central and peripheral autonomic nervous system has an important role in carrying the circadian signals from the SCN clock to the peripheral organs (Buijs and Kalsbeek, 2001; Terazono et al., 2003; Ishida et al., 2005).

In the present study, we investigated how circadian rhythmicity is conducted to the respiratory system. We first examined the basic features of clock system in the respiratory tract including larynx, trachea, bronchus, and lung, focusing on the circadian regulation of clock genes. Next, we investigated the role of acetylcholine neurotransmission in the circadian change of respiratory functions, using vagotomized and arrhythmic Cry $1^{-1-} \mathrm{Cr} y 2^{-/-}$ mice. Our data for the first time show that the vagal nerve conveys rhythmic signals.

\section{Materials and Methods}

Animals. Adult wild-type C57BL/6 (Japan Animals, Osaka, Japan) and Cry $1^{-1-}$ Cry $2^{-1-}$ mice (van der Horst et al., 1999) were housed for at least 2 weeks in an environment with a $12 \mathrm{~h}$ light/dark cycle with food and water ad libitum, and subsequently moved to constant darkness (DD). All animal experiments were approved by the Committee for Animal Research of Kobe University.

Vagotomy and sympathectomy. Unilateral cervical vagotomy and unilateral and bilateral sympathectomy were performed under ketamine/ xylazine anesthesia. For cervical vagotomy $(n=18)$, the right cervical vagal nerve was cut at the level of the jugular foramen. For shamoperated animals $(n=8)$, the vagal nerves were similarly exposed, but 
A

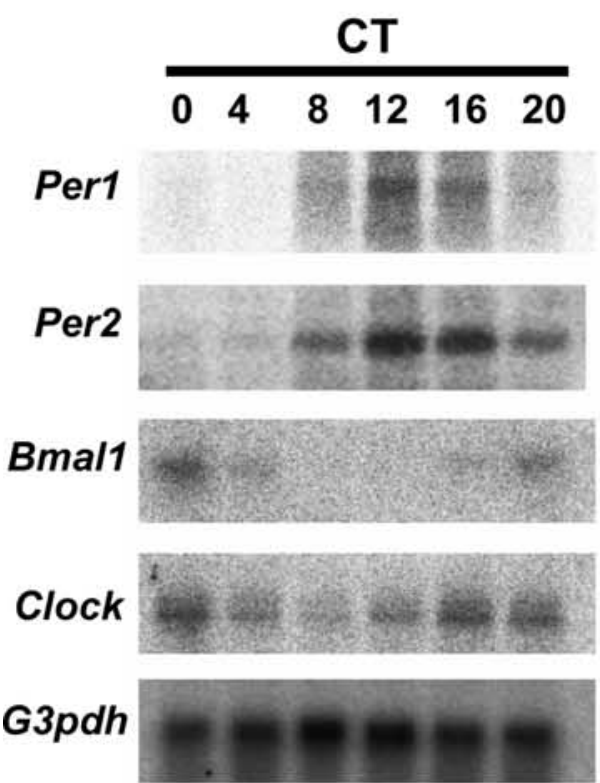

E
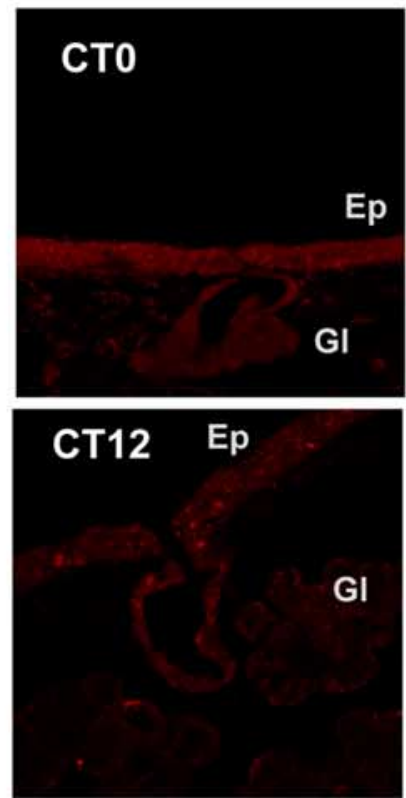

B

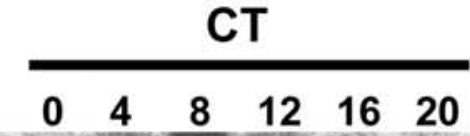

Dbp

G3pdh

D
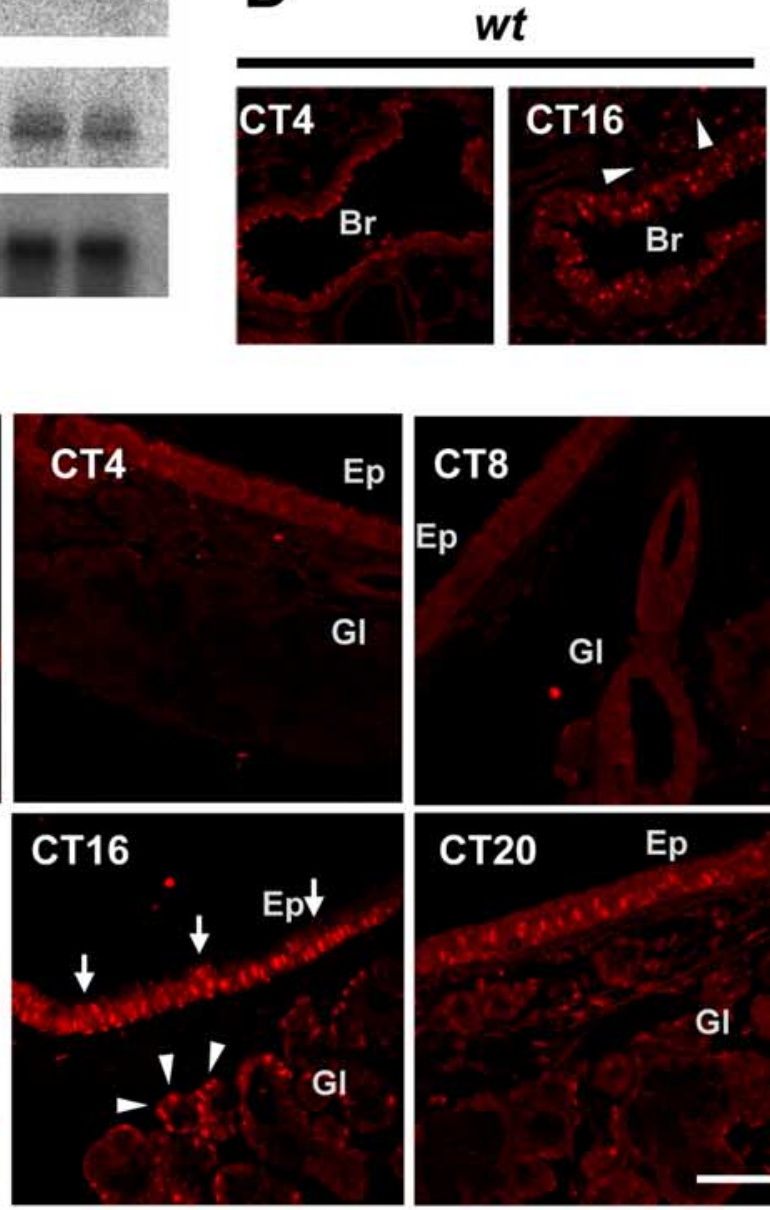
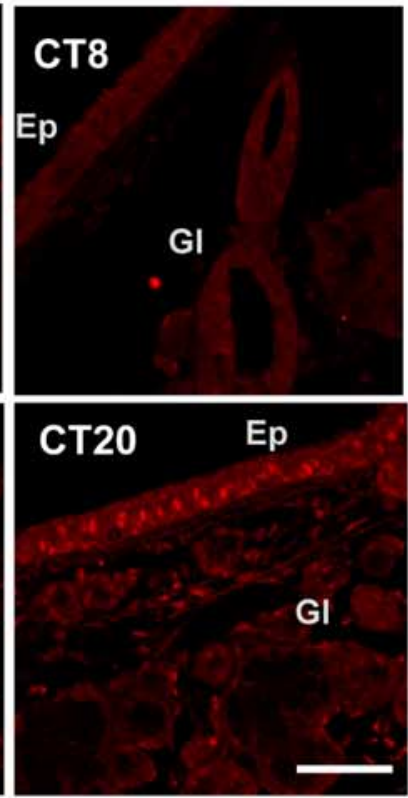

C

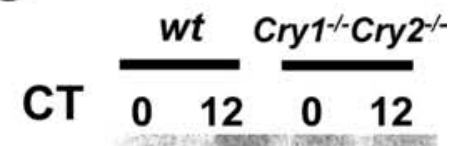

Dbp

G3pdh

Cry1\%-Cry2\%-

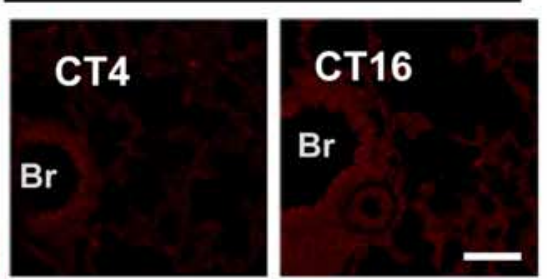

F

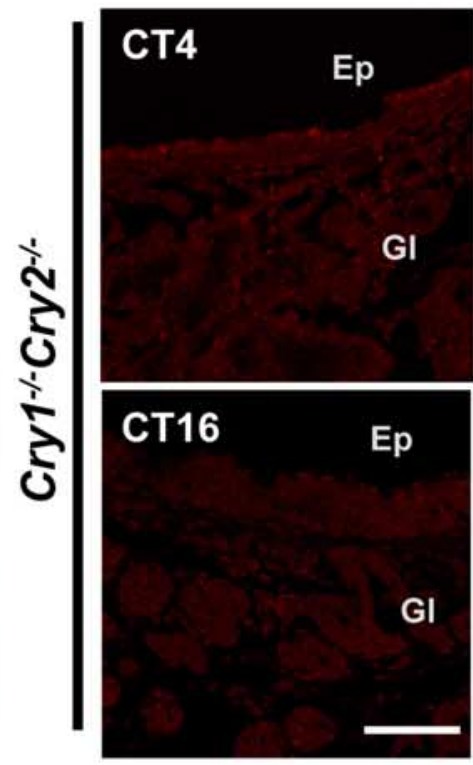

G SCN-lesion Time of Day

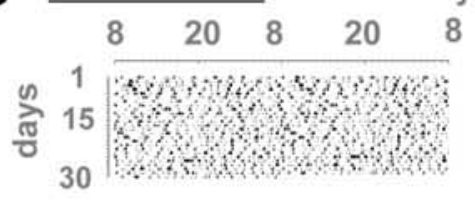

\section{Sham-ope Time of Day}

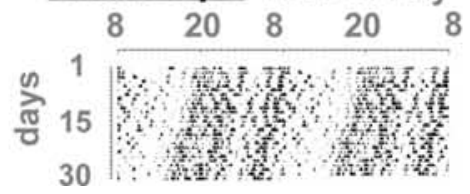

Sham- SCNoperated lesioned

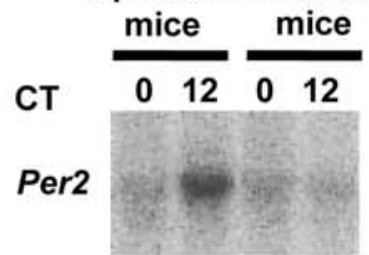

G3pdh

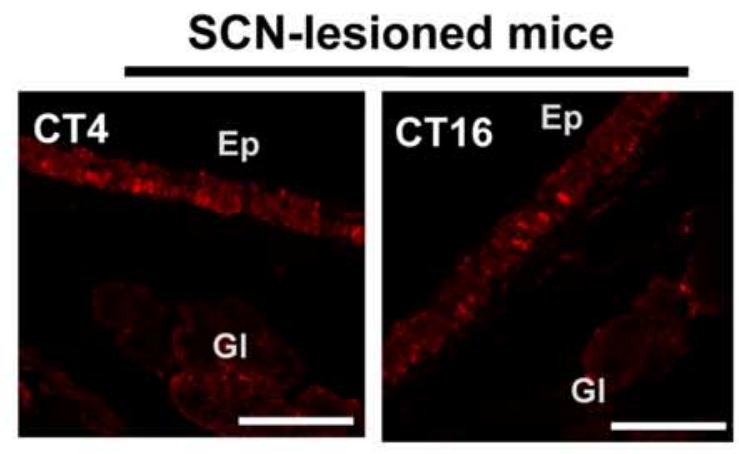

Figure 1. Clock genes and clock controlled genes in the mouse respiratory system. $\boldsymbol{A}$, Circadian expression of Per1, Per2, Bmal1, and Clock genes, as detected by Northern blot analysis. G3pdh expression was determined as a control. Note the peak and through in Per 1 and Per2 mRNA levels at CT12 and CT0, respectively, as well as the inverted rhythms of Bmal1 and Clock gene expression. $\boldsymbol{B}$, Circadian expression of Dbp, a clock controlled gene. Dbp mRNA levels show a similar distribution pattern as that of Per1. C, Dbp mRNA levels in arrhythmic (Figure legend continues) 
not cut. Cervical sympathectomy was done by extirpation of the right side $(n=4)$, or both sides $(n=6)$ of the cervical sympathetic trunk, including the superior and middle cervical ganglia. Hemivagotomized mice were killed at CT2 [circadian time (CT), CT0, subjective dawn; CT12, subjective dusk] $(n=3)$, CT4 $(n=8)$, CT6 $(n=3)$, CT14 $(n=3)$, CT16 $(n=8)$, and CT18 $(n=3)$ for histological analysis.

SCN lesioning and locomotor activity. Bilateral thermal electrical lesioning of the SCN was performed under ketamine/hydrazine anesthesia $(n=12)$ using a stereotactic microscope. A lesion was made by passing a current of $15 \mathrm{~mA}$ for $15 \mathrm{~s}$. Sham-operated animals $(n=6)$ were subjected to the same procedure except that the current path was omitted. Complete lesioning of the SCN was confirmed by checking the locomotor activity using passive infrared sensors and histological sections of the hypothalamus.

Histology and immunohistochemistry. Larynx, trachea, bronchus, and lung were processed for immunocytochemistry with a rabbit antimPER2 antiserum (1:500; Alpha Diagnostic, San Antonio, TX) or a goat anti-MUC5B antiserum (1:1000; Santa Cruz Biotechnology, Santa Cruz, $\mathrm{CA})$. The primary antibodies were visualized using a Cy3-conjugated anti-rabbit IgG (1:500; Vector Laboratories, Burlingame, CA) or an FITC-conjugated anti-goat IgG (1:500; Vector Laboratories), and Zeiss (Oberkochen, Germany) confocal microscopy. Nuclei were visualized by DAPI (4,6,diamidino-2-phenylindole) staining. Statistical analysis of the number of PER2-positive nuclei in epithelial and glandular cells of the trachea was performed after vagotomy at CT4 and CT16, and analyzed by Student's $t$ test $(n=3)$.

Mucin was visualized by Alcian blue (AB) and periodic acid-Schiff (PAS) staining of acidic and neutral intracellular mucins, respectively. Hematoxylin was used for counterstaining.

Northern blot analysis. Northern blot analysis was performed as described previously (Ishida et al., 2005). Samples were collected on the second day in DD at CT0, CT4, CT8, CT12, CT16, and CT20 $(n=4$ at each time point). For elucidating the effect of deletion of circadian clock, Cry $1^{-1-}$ Cry $2^{-1-}$ and wild-type mice were sampled at CT4 and CT16 ( $n=8$ at each time point). ${ }^{32} \mathrm{P}$-radiolabeled probes were produced from the following reverse transcription-PCR-derived fragments: Per1 (16383373, GenBank accession number AF022992), Per2 (1-636, AF035830), Bmal1 (663-1482, AB000812), Dbp (595-1100, U29762), Chrm1 (30503569 NM_007698), Chrm2 (604-1177, NM_203491), Chrm3 (2419-2960, NM_033269), Chrm4 (629-1020, NM_007699). For glyceraldehyde 3-phosphate dehydrogenase (G3pdh) mRNA detection, we used the Human G3PDH control probe (Clontech, Mountain View, CA).

Real-time PCR. mRNA levels were quantified by real-time PCR analysis (ABI Prism 7700 Sequence Detection system; Applied Biosystems, Foster City, CA). Oligonucleotide primers and TaqMan probes for Chrm1, Chrm2, Chrm3, Chrm4, and G3pdh were ordered as kits (TaqMan Gene Expression Assays; Applied Biosystems). All samples were amplified simultaneously in triplicate in one assay run. G3pdh was used as a control. For all measurements, mRNA levels are expressed as mean \pm SEM. Differences in mRNA levels between groups were analyzed by oneway ANOVA, followed by Fisher's least protected difference test. Significance was set at a value of $p<0.05$.

In situ hybridization. Frozen or paraffin-embedded laryngeal sections were pretreated with proteinase $\mathrm{K}$ and $0.25 \%$ acetic anhydride/triethanolamine, and hybridized with antisense Muc5b probes (14101-14658, NM_028801) labeled with digoxigenin-dUTP using the DIG-tailing Kit
A
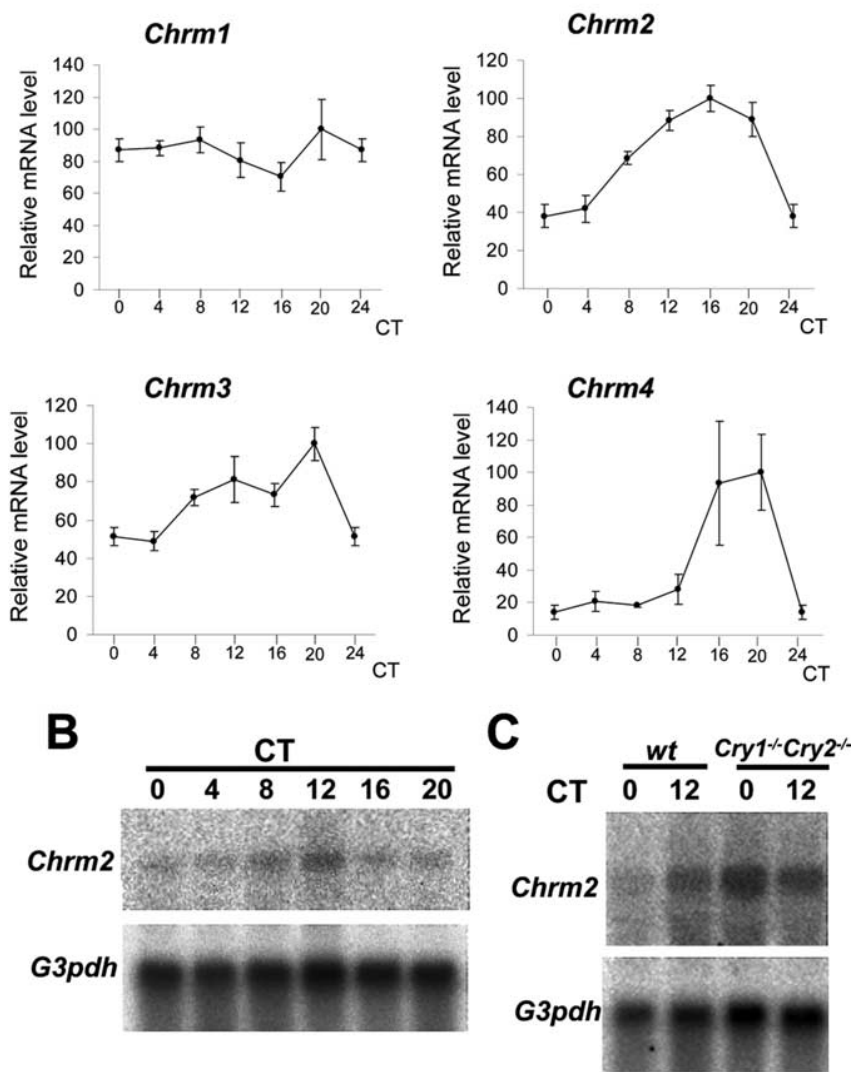

Figure 2. The circadian gene expression profiles of Chrm1, Chrm2, Chrm3, and Chrm4. A, Quantitative real-time PCR analysis of muscarinic acetylcholine receptor (Chrm1-4) gene expression in the lung of $\mathrm{C57BL} / 6$ mice at various circadian times. G3pdh expression was determined as a control. Whereas Chrm $1 \mathrm{mRNA}$ levels did not oscillate in a circadian manner, Chrm2, Chrm3, and Chrm4 transcription showed significant circadian rhythmicity, peaking at CT16CT20. B, Northern blot analysis of Chrm2 mRNA levels in the lung of C57BL/6 mice, showing clear circadian rhythmicity. C, Northern blot analysis of Chrm2 expression in the lungs of $\mathrm{Cry}^{-/-}$ $\mathrm{Cry}^{-/-}$mice and wild-type littermates at CT0 and CT12. Note the constant high Chrm2 mRNA levels in $\mathrm{Cry}^{-/-}$Cry $2^{-/-}$mice.

(Boehringer Mannheim, Mannheim, Germany). Negative controls included a sense probe and excess (100-fold) unlabeled probe.

\section{Results}

\section{Respiratory organs contain an active peripheral circadian} core oscillator

We first examined circadian clock gene expression in the respiratory organs (including the larynx, trachea, bronchus, and lung) of wild-type and circadian clock-deficient $\mathrm{Cry} 1^{-/-} \mathrm{Cry} 2^{-/-}$mice. Unless specified otherwise, animals were kept in constant dark to eliminate the effect of environmental light.

Northern blot analysis of Per1 and Per2 expression in the lung of wild-type mice revealed significant circadian rhythmicity with

$\mathrm{Cry} 1^{-/-} \mathrm{Cry} 2^{-/-}$mice. D, Immunohistochemical staining of the PER2 protein in the lung and bronchiole of wild-type and $\mathrm{Cry} 1^{-/-} \mathrm{Cry} 2^{-/-}$mice at CT4 and CT16. PER2 proteins were abundantly expressed in the nucleus of bronchial epithelial cells at CT16, although almost absent at CT4. Alveolar cells (arrowheads) also showed a slight rhythm. PER2 protein expression was abolished in $C r y 1^{-1-}$ Cry $2^{-1-}$ mice at any time examined. $\boldsymbol{E}$, Circadian expression of the PER2 protein in epithelium and submucosal glands in trachea. Note the high level of nuclear immunohistochemical staining in tracheal epithelium (arrows) and submucosal glands (arrowheads) at CT16. F, Abolished PER2 protein expression in Cry $1^{-/-}$Cry2 ${ }^{-1-}$ mice. G, Loss of circadian oscillation in Per2 mRNA and PER2 protein levels in the respiratory system of SCN-lesioned animals. Shown are (double-plotted) behavioral actograms (left), and Per2 mRNA expression levels at CT0 and CT12, as determined by Northern blot analysis (middle) of SCN-lesioned and sham-operated wild-type mice, as well as PER2-immunohistochemistry in SCN-lesioned mice at CT4 and CT16. Data were adopted as control versus sham-operated animals. Br, Bronchiole; Ep, tracheal epithelium; Gl, submucosal gland. Scale bars, $100 \mu \mathrm{m}$. 


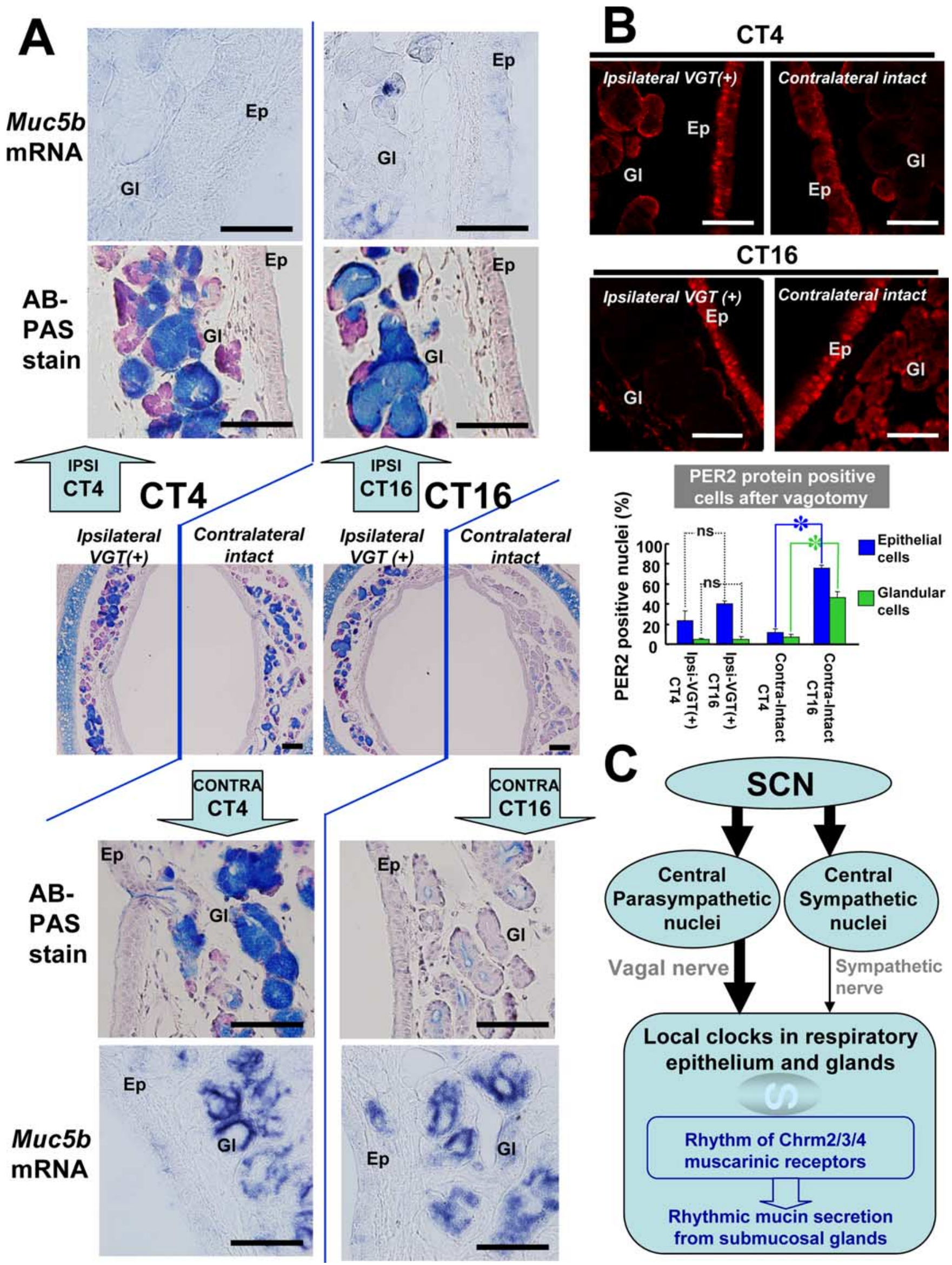


a peak at CT12-CT16, and a trough at CT0-CT4 (Fig. 1A). In contrast, Bmall and Clock mRNA levels showed peaks at CT20CT24(CT0) and troughs at CT8-CT12. The antiphase expression patterns of Per1/Per2 and Bmall/Clock genes demonstrate the presence of a functional peripheral circadian core oscillator in the lung. We then examined whether this core clock oscillation effectively drives the cyclic transcription of downstream genes. As an indicator of clock output, we determined the expression profiles of the $D b p$ gene, which is directly regulated by clock genes (Yamaguchi et al., 2000). Dbp mRNA levels showed a peak at CT12-CT16 and a trough at CT4 in wild-type mice (Fig. $1 B$ ), but rhythmicity was completely abolished in arrhythmic $\mathrm{Cry}^{-/-} \mathrm{Cry} 2^{-/-}$mice (Fig. 1C). This finding strongly indicates that the peripheral core oscillator in the lung effectively drives cyclic expression of clock output genes.

Next, we determined which cell type is responsible for the observed circadian expression patterns of core clock and output genes in the lung. Immunohistochemical staining of the PER2 protein in lung sections of animals killed at CT16 revealed strong PER2 expression in nuclei of the pseudocolumnar respiratory ciliary epithelial cells of the bronchiole (Fig. 1D). At this time point, alveolar cells also showed a moderate level of PER2. In contrast, the PER2 protein was barely detectable in both bronchoepithelial and alveolar cells at CT4. In arrhythmic Cry $1^{-/-}$Cry $2^{-/-}$mice, PER2 protein was always absent in epithelial and alveolar cells at both CT4 and CT16.

We next focused on the upper respiratory tract and performed a detailed immunohistochemical analysis of circadian expression profiles in the larynx and trachea. PER2 immunoreactivity showed a significant circadian rhythm in the epithelium, as well as in the submucosal gland (present in the lower larynx and continuing in the trachea), with a peak at CT16. As shown in Figure $1 E$, nuclear staining of PER2 was most pronounced in the acinar and ductal cells in the submucosal glands (arrowheads), as well as in respiratory ciliary epithelial cells (arrows) at CT16 and CT20. In contrast, the PER2 protein was never observed at CT0, CT4, and CT8, and only present in few epithelial at CT12. Submucosal glandular cells in the larynx to trachea also showed a clear PER2 protein rhythm with a peak at CT16 and a trough at CT0-CT4. As expected, PER2 protein could not be detected in epithelial and glandular cells of arrhythmic $\mathrm{Cr} y 1^{-/-} \mathrm{Cry} 2^{-/-}$mice at all time points examined (Fig. $1 F$ ). These findings demonstrated that core clock oscillation occurs in all cells of the lung and upper respiratory tract (i.e., larynx and trachea).

To address the question whether the peripheral clocks in the respiratory organs are driven by the master clock in the SCN, we ablated the SCN of wild-type mice by electric coagulation. Analysis of locomotor behavior confirmed the complete loss of rhythmicity in SCN-lesioned animals, as well as the intactness of the master clock in sham-operated animals, used as a control. North- ern blot analysis of Per2 mRNA levels revealed that SCNlesioning eradicates rhythmic expression of this clock gene in the lungs (Fig. 1G, middle). Moreover, by the analysis of immunohistochemistry, the PER2 staining was always (CT4 and CT16) absent or very rare in the glandular cells and was always faint in the epithelial cells in SCN-lesioned mice (Fig. 1G, right), although a clear rhythm in PER2 protein expression was detected in epithelium and glands in sham-operated controls (data not shown). This finding demonstrates that peripheral clock oscillations in the respiratory tract are driven by the master circadian clock in the SCN.

\section{The expression of Chrm 2, 3, and 4 muscarinic receptors is regulated by the circadian clock}

Having shown that the respiratory system contains a peripheral circadian oscillator, we next focused on clock output genes. Because at present knowledge on circadian output genes is limited, we concentrated on physiological processes in the respiratory system that are known to display circadian characteristics. Because it is known that pharmacological agents relating to the muscarinic acetylcholine receptor have a strong effect on airway contraction and mucin secretion in respiratory system (Barnes, 1993), we started to examine the mRNA expression profiles of various subtypes of muscarinic receptor genes $(\mathrm{Chrm} 1-4)$ that are known to be expressed in the lung (Barnes, 1993; Ramnarine et al., 1996). Although quantitative real-time PCR analysis did not show circadian variation in Chrm 1 mRNA levels (one-way ANOVA; $\left.F_{(5,23)}=1.040 ; p=0.4180\right)$, we noticed a significant circadian rhythm in Chrm 2 expression (one-way ANOVA; $F_{(5,24)}=$ 15.992; $p<0.0001$ ), peaking at CT16 with a 2.63-fold amplitude (Fig. 2A). This finding is also supported by Northern blot analysis, revealing cycling Chrm 2 mRNA levels with a peak at CT12 and a trough at CT0-CT4 (Fig. 2B). Chrm3 mRNA also shows rhythmic expression (one-way ANOVA; $F_{(5,24)}=7.016$; $p<0.001$ ), with mRNA levels being higher at subjective dusk than at subjective morning (Fig. 2A). Although the expression level of Chrm 4 was the lowest among other muscarinic receptors $(\mathrm{m} 2>\mathrm{m} 3>\mathrm{m} 1>\mathrm{m} 4)$, mRNA levels showed a clear circadian rhythm (one-way ANOVA; $F_{(5,24)}=4.399 ; p<0.01$ ) with a peak in the subjective evening and a trough in the subjective morning (Fig. 2A). Chrm2, Chrm3, and Chrm4 oscillations were not observed in $\mathrm{Cry}^{-/-} \mathrm{Cry} 2^{-/-}$mice (Fig. 2C, data not shown), indicating that transcription of these genes is directly and/or indirectly regulated by the circadian core clock genes. Because these three subtypes of molecules are main components of muscarinic transmission in the lung, it is speculated that there may be circadian modulation of acetylcholine neurotransmission at the receptor level.

Figure 3. Vagotomy-induced changes in mucin levels and clock gene expression. A, Mucin levels (AB/PAS) and Muc5b gene expression (in situ hybridization) at CT4 and CT16 in the trachea of C57BL/6 mice, one week after hemivagotomy. Middle, Low-magnification photographs of the AB/PAS-stained trachea, showing the ipsilateral vagotomized [VGT(+)] side and the contralateral intact side. Top, bottom, High-magnification photographs of the AB/PAS-stained ipsilateral vagotomized side and contralateral intact side, respectively. In addition, Muc $5 b$ mRNA levels (as determined by in situ hybridization) at the ipsilateral side (top panels) and the contralateral intact side (bottom panels) are shown at high magnification. $\boldsymbol{B}$, Tracheal PER2 protein after vagotomy at CT4 and CT16. Br, Bronchiole; Ep, tracheal epithelium; Gl, submucosal gland. The number of PER2 protein-positive nuclei in epithelial cells and glandular cells in trachea was calculated in the ipsilateral vagotomized side at CT4 (Ipsi-VGT(+)-CT4), ipsilateral vagotomized side at CT16 [Ipsi-VGT(+)-CT16], contralateral intact side at CT4 (Contra-Intact-CT4), and contralateral intact side at CT16 (Contra-Intact-CT16). Note the absence of rhythmic mPER2 expression at the vagotomized side, in contrast to the clear rhythm in contralateral intact side in both epithelial and glandular cells. Statistics was performed by Student's $t$ test (mean \pm SEM; $n=3 ;{ }^{*} p<0.05$ ). ns, Not significant. C, Model for the circadian control of mucin secretion in respiratory organs. Circadian expression of the muscarinic cholinergic receptor genes Chrm2, Chrm3, and Chrm 4 is under control of the local peripheral circadian clock. The peripheral oscillator receives input from the central circadian oscillator in the SCN via the autonomous nervous system, in which vagal innervation is highly dominant over sympathetic innervation. Mucin production is constant, but the release of this glycoconjugate from the submucosal gland is increased at night time via increased muscarinic receptor expression and increased vagal tone. Scale bars: $100 \mu \mathrm{m}$. 


\section{The vagal nerve transmits circadian signal to glandular mucus secretion}

It is thought that the central and peripheral autonomic nervous systems carry circadian output signals from the SCN master clock to the periphery (Buijs and Kalsbeek, 2001; Terazono et al., 2003; Ishida et al., 2005). The respiratory tract is innervated mainly by parasympathetic (vagal) nerves (Nadel and Barnes, 1984). To examine the involvement of the vagal nerve in the respiratory system, we performed unilateral vagotomy (right vagus cut) and examined the circadian expression profiles of clock genes as well as mucin synthesis in the ipsilateral (lesioned; right) and contralateral (unlesioned; left) sides of the larynx and trachea (Fig. 3).

We first applied a routine histological staining (AB/PAS) for detecting mucous glycoconjugates in the submucosal gland (Fig. $3 A$ ). Interestingly, on the contralateral intact side, we observed dense $\mathrm{AB} / \mathrm{PAS}$ staining of tracheal glands at subjective day time (CT2, CT4, and CT6), whereas staining was weak at the subjective night (CT14, CT16, and CT18) (Fig. 3A, middle), thus pointing to the presence of a circadian control mechanism. At the vagotomized side, however, we observed very dense AB/PAS staining of the glands (compared with sham-operated animals) at any time examined. On the contrary, both ipsilateral and bilateral sympathectomy did not affect the AB/PAS staining patterns (data not shown). Thus, we conclude that the vagal nerve, rather than the sympathetic nerve, mediates the crucial information that controls circadian oscillations in glandular mucous glycoconjugate levels.

Because the intensity of $\mathrm{AB} / \mathrm{PAS}$ staining in the glandular cells is determined by the net result of production and release from its cells, we cannot discriminate whether the oscillation in AB/PAS staining at the contralateral side is controlled by rhythms in mucous glycoconjugate production, or by rhythmic release of mucins by the glandular cell, and whether the constant high levels of this compound at the ipsilateral side are caused by loss of rhythmic synthesis (now being constitutively high) or release. To address this issue, we next examined the expression level of the $M u c 5 b$ gene, encoding the main subtype of mucin produced by the submucosal gland (Chen et al., 2001). Importantly, Muc5b mRNA levels at the nonvagotomized contralateral side did not oscillate (Fig. 3A, bottom), indicating that the production of mucin is constant. Nevertheless, in line with the rhythm in AB/PAS staining, intracellular MUC5B protein levels show a diurnal rhythm in the submucosal gland (data not shown). Despite high intensity $\mathrm{AB} / \mathrm{PAS}$ staining patterns, we noticed the severe reduction of $M u c 5 b$ mRNA levels at the vagotomized ipsilateral side regardless of subjective day and night (Fig. $3 A$, head panel). Together, these findings suggest that mucin release, rather than synthesis, is influenced by circadian time-information received through the vagal nerve, with enhanced secretion at the night time.

The autonomic control of the tracheal clock was directly examined by immunohistochemical staining of the PER2 clock protein. Because ipsilateral and bilateral sympathectomized mice did not show alterations in PER2 expression patterns in both tracheal epithelium and gland (data not shown), it is speculated that the sympathetic nerves have little effect on the transmission of time-information. In the ipsilateral side of the vagotomized mice, however, fewer nuclei were positive for PER2 expression in the epithelial cells and glandular cells at both CT4 and CT16 (Fig. $3 B$ ). The pattern of reduction in the vagotomized side mimics that after the SCN-lesioning (compare Figs. $1 G, 3 B$ ). In the contralateral side, PER2 protein was abundantly observed in the nu- clei of epithelial cells and submucosal glandular cells at CT16, but nothing at CT4 (Fig. 3B), which rhythm is similar to be observed in the intact mice (compare Fig. $3 B$ with Fig. $1 E$ ). This finding indicates that the glandular and epithelial cells receive nervous input from the ipsilateral side, and that the vagal nerve is crucial for transmitting time signals from the SCN to the glandular and epithelial cells.

\section{Discussion}

In the present study, we have demonstrated the presence of an active circadian core oscillator in cells of the respiratory tract: larynx, trachea, bronchus, and lung. There, Per1 and Per 2 mRNA levels show a robust rhythm, accompanied by antiphase oscillations in Bmall and Clock mRNA levels. This expression pattern is common to all clock works in the body, although the $8 \mathrm{~h}$ phase delay of the peripheral respiratory tract oscillator with the SCN master oscillator is very similar to that observed in other visceral organs (Damiola et al., 2000). The finding that SCN lesioning abolished all of the peripheral clock work in the respiratory gland confirmed the master role of the SCN clock on the respiratory peripheral clock. This finding is in complete concordance with previous transplantation studies showing the slave properties of peripheral clocks to the master oscillation of the SCN (Pando et al., 2002; Sujino et al., 2003). The presence of a peripheral circadian oscillator in the respiratory tract also explains a previous report, showing abolished rhythms of oxygen consumption rate and respiratory quotient values after lesioning of the SCN lesion in the rat brain (Nagai et al., 1985).

Pulmonary functions such as forced expiratory volume and peak expiratory flow show diurnal variation (Barnes et al., 1985). Moreover, important symptoms of asthmatic attacks such as wheezing (caused by the contraction of airways) and increased mucus secretion peak in the late night/early morning. Although several factors (including mast cells, circulating adrenaline, and corticosterone) are involved, it is proposed that an increase in the vagal tone at night may trigger these pathophysiological events (Barnes, 1985). Our present findings strongly suggest that mucin secretion, but not synthesis, is under the control of the circadian system. Circadian signals from the master circadian clock in the SCN have been shown to convey to peripheral organs via two neural routes: central sympathetic (e.g., intermediolateral cell column) and central parasympathetic (e.g., dorsal motor nucleus of the vagus and the region nearby the nucleus ambiguus) innervation (Buijs and Kalsbeek, 2001) (Fig. 3C). The respiratory tract is innervated by both sympathetic and parasympathetic nerves, but vagal innervation overwhelms sympathetic innervation in bronchial smooth muscles, airway ganglia and submucosal glands (Nadel and Barnes, 1984). In the present study, we found that hemivagotomy completely suppressed clock gene expression in the ipsilateral submucosal glandular cells, and moderately suppressed the peripheral oscillator in epithelial cells. In marked contrast, cyclic clock gene expression was not suppressed after sympathectomy. Because SCN lesion completely abolished the respiratory rhythm, these findings strongly indicate that the vagal nerve is the dominant route for conveying circadian time signals from the SCN master clock to the peripheral clock in the respiratory tract.

Interestingly, after hemivagotomy, we found an increase in mucin secretion at the intact contralateral side. In a separate experience, we also found that the circadian clock regulates expression of the muscarinic acetylcholine receptors; expression levels being high in the subjective night and low in the subjective day. Thus, the increased nocturnal mucin secretion may be explained 
by an increase in muscarinic receptors in conjunction with an enhanced vagal tone at night. Because it has been demonstrated that cholinergic nerve stimulation promotes mucus secretion via Chrm3 receptors in the ferret trachea (Ramnarine et al., 1996), a circadian raise in Chrm 3 receptor levels may trigger the enhanced mucus secretion during night time. The circadian change of the acetylcholine neurotransmission, as revealed in this study, extends our understanding of the pathophysiology of asthmatic symptoms and airway diseases, and may help to improve therapeutic strategies.

\section{References}

Barnes PJ (1985) Circadian variation in airway function. Am J Med 79:5-9. Barnes PJ (1993) Muscarinic receptor subtypes in airways. Life Sci 52:521-527.

Buijs RM, Kalsbeek A (2001) Hypothalamic integration of central and peripheral clocks. Nat Rev Neurosci 2:521-526.

Chen Y, Zhao YH, Wu R (2001) In silico cloning of mouse Muc5b gene and upregulation of its expression in mouse asthma model. Am J Respir Cirt Care Med 164:1059-1066.

Damiola F, Minh N, Prenitner N, Kornmann B, Fleury-Olela F, Schibler U (2000) Restricted feeding uncouples circadian oscillators in peripheral tissues from the central pacemaker in the suprachiasmatic nucleus. Genes Develop 14:2950-2961.

Hastings MH, Reddy AB, Maywood ES (2003) A clockwork web: circadian timing in brain and periphery, in health and disease. Nat Rev Neurosci 4:649-661.

Ishida A, Mutoh T, Ueyama T, Bando H, Masubuchi S, Nakahara D, Tsuji- moto G, Okamura H (2005) Light activates the adrenal gland: timing of gene expression and glucocorticoid release. Cell Metab 2:297-307.

Nadel JA, Barnes PJ (1984) Autonomic regulation of the airways. Annu Rev Med 35:451-467.

Nagai K, Nishio T, Nakagawa H (1985) Bilateral lesions of suprachiasmatic nucleus eliminate circadian rhythms of oxygen consumption and the respiratory quotient in rats. Experientia 41:1136-1138.

Pando MP, Morse D, Cermakian N, Sassone-Corsi P (2002) Phenotypic rescue of a peripheral clock genetic defect via SCN hierarchical dominance. Cell 110:107-117.

Ramnarine SI, Haddad EB, Khawaja AM, Mak JC, Rogers DF (1996) On muscarinic control of neurogenic mucus secretion in ferret trachea. J Physiol (Lond) 494:577-586.

Schibler U, Sassone-Corsi P (2002) A web of circadian pacemakers. Cell 111:919-922.

Sujino M, Matsumoto K, Yamaguchi S, van der Horst G, Okamura H, Inouye SIT (2003) Suprachiasmatic nucleus grafts restore circadian behavioral rhythms of genetically arrhythmic mice. Curr Biol 13:664-668.

Terazono H, Mutoh T, Yamaguchi S, Kobayashi M, Akiyama M, Udo R, Ohdo S, Okamura H, Shibata S (2003) Adrenergic regulation of clock gene expression in the mouse liver. Proc Natl Acad Sci USA 100:6795-6800.

van der Horst GTJ, Muijtjens M, Kobayashi K, Takano R, Kanno S, Takao M, de Wit J, Verkerk A, Eker APM, von Leenen D, Buijs R, Bootsma D, Hoeijmakers JHJ, Yasui A (1999) Mammalian Cry1 and Cry2 are essential for maintenance of circadian rhythms. Nature 398:627-630.

Yamaguchi S, Mitsui S, Yan L, Yagita K, Miyake S, Okamura H (2000) Role of DBP in the circadian oscillatory mechanism. Mol Cell Biol 20:4773-4781. 